Supplement of Geosci. Model Dev., 13, 4271-4285, 2020

https://doi.org/10.5194/gmd-13-4271-2020-supplement

(C) Author(s) 2020. This work is distributed under

the Creative Commons Attribution 4.0 License.

(c) (1)

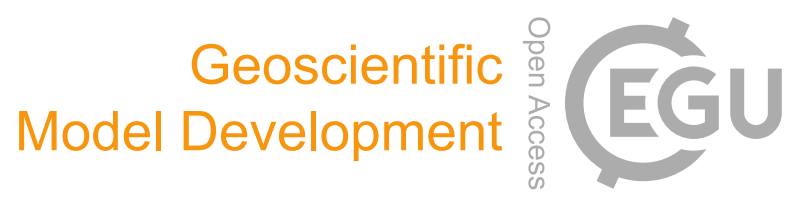

Supplement of

\title{
Can machine learning improve the model representation of turbulent kinetic energy dissipation rate in the boundary layer for complex terrain?
}

Nicola Bodini et al.

Correspondence to: Nicola Bodini (nicola.bodini@nrel.gov)

The copyright of individual parts of the supplement might differ from the CC BY 4.0 License. 


\section{Proof on approximation for MYNN parameterization of TKE dissipation rate}

Actual definition:

Approximation:

$$
\frac{1}{L_{M}}=\frac{1}{L_{S}}+\frac{1}{L_{T}}+\frac{1}{L_{B}}
$$

$$
\frac{1}{L_{M}} \approx \frac{1}{L_{S}}
$$

Thesis: the approximation will always overestimate $L_{M}$

Proof:

Let's compare the right-hand sides of the definition of $L_{M}$ and the approximation followed in our analysis and suppose our thesis is true. Also, all length scales are strictly positive.

$$
\begin{gathered}
\left(\frac{1}{L_{S}}+\frac{1}{L_{T}}+\frac{1}{L_{B}}\right)^{-1}<L_{S} \\
\frac{L_{S} L_{T} L_{B}}{L_{T} L_{B}+L_{S} L_{B}+L_{S} L_{T}}<L_{S} \\
\frac{L_{T} L_{B}}{L_{T} L_{B}+L_{S} L_{B}+L_{S} L_{T}}<1 \\
L_{T} L_{B}<L_{T} L_{B}+L_{S} L_{B}+L_{S} L_{T} \\
0<L_{S} L_{B}+L_{S} L_{T}
\end{gathered}
$$

Which is always true since all length scales are strictly positive. 
2. Bias in MYNN parameterization using data from the three 100m met towers

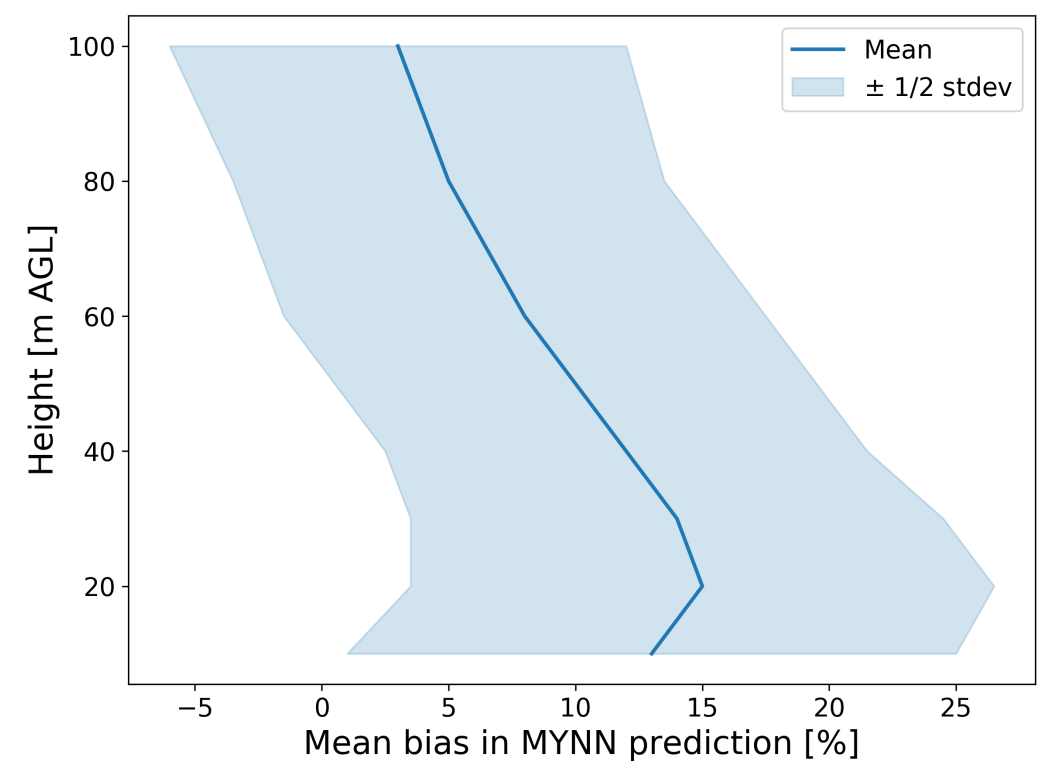

Figure S1: Mean bias in the MYNN-parameterized $\log \epsilon$ at different heights, as calculated from the sonic anemometers on the three 100-m towers at Perdigão. 


\section{Input and output features distributions}
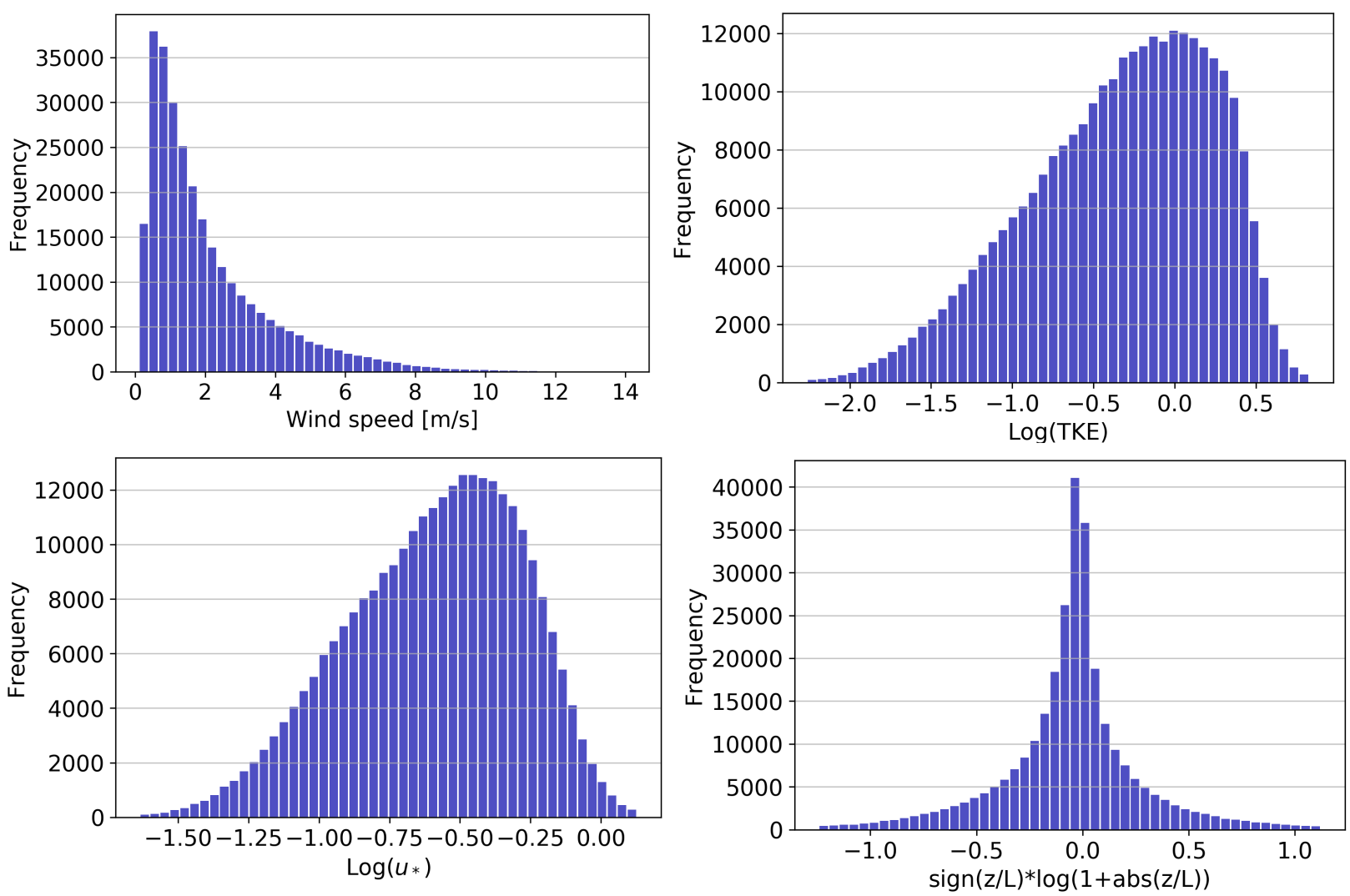

Figure S2: Distributions of 30-minute average wind speed, $\log (T K E), \log \left(u_{*}\right)$, and $\operatorname{sign}(z / L) \log (1+a b s(z / L))$ from the sonic anemometers at Perdigão. 

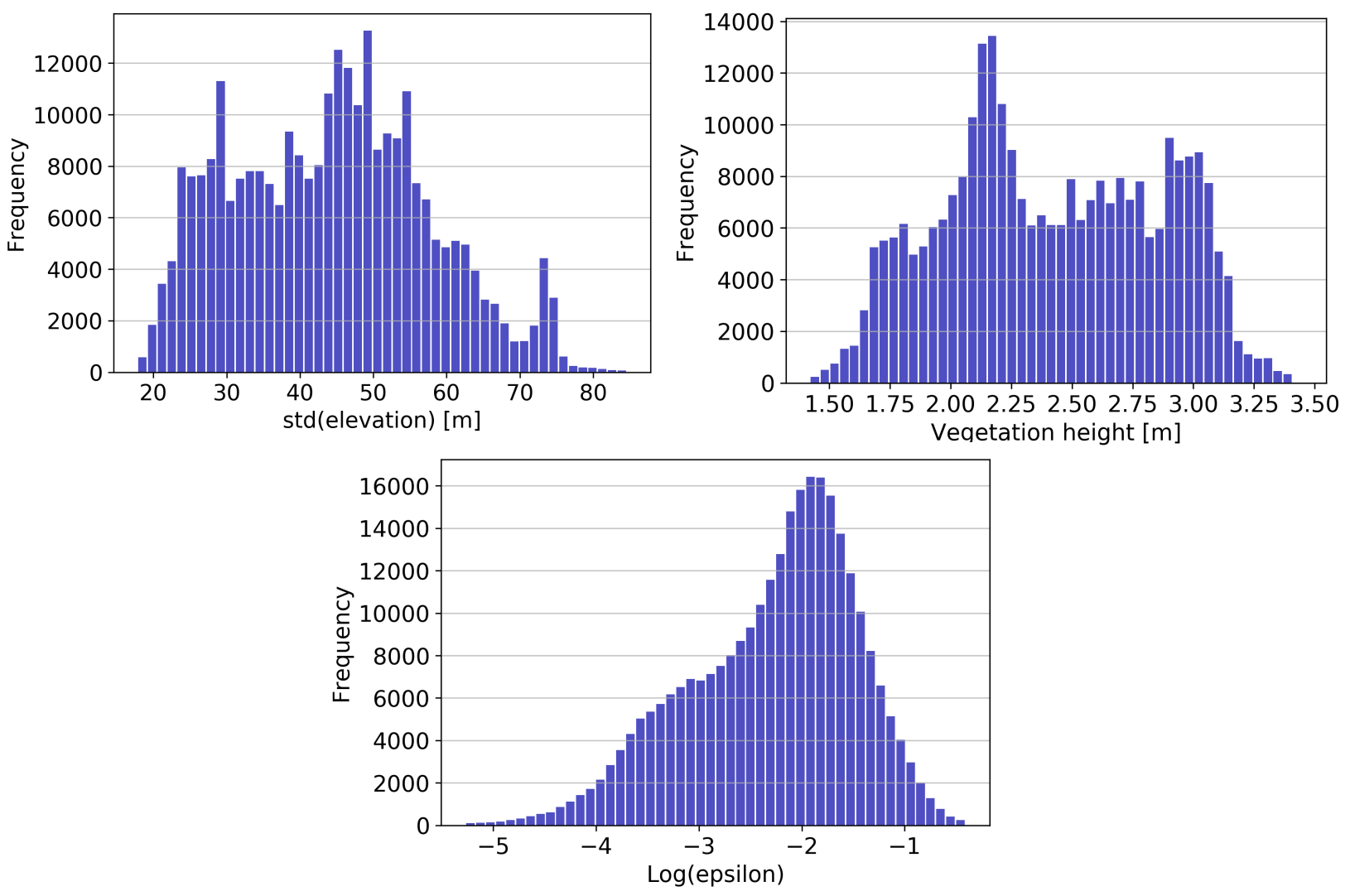

Figure S3: Distributions of $\sigma\left(z_{\text {terr }}\right)$, vegetation height, and 30-minute average $\log \epsilon$ from the sonic anemometers at Perdigão. 


\section{Feature importance as a function of height}

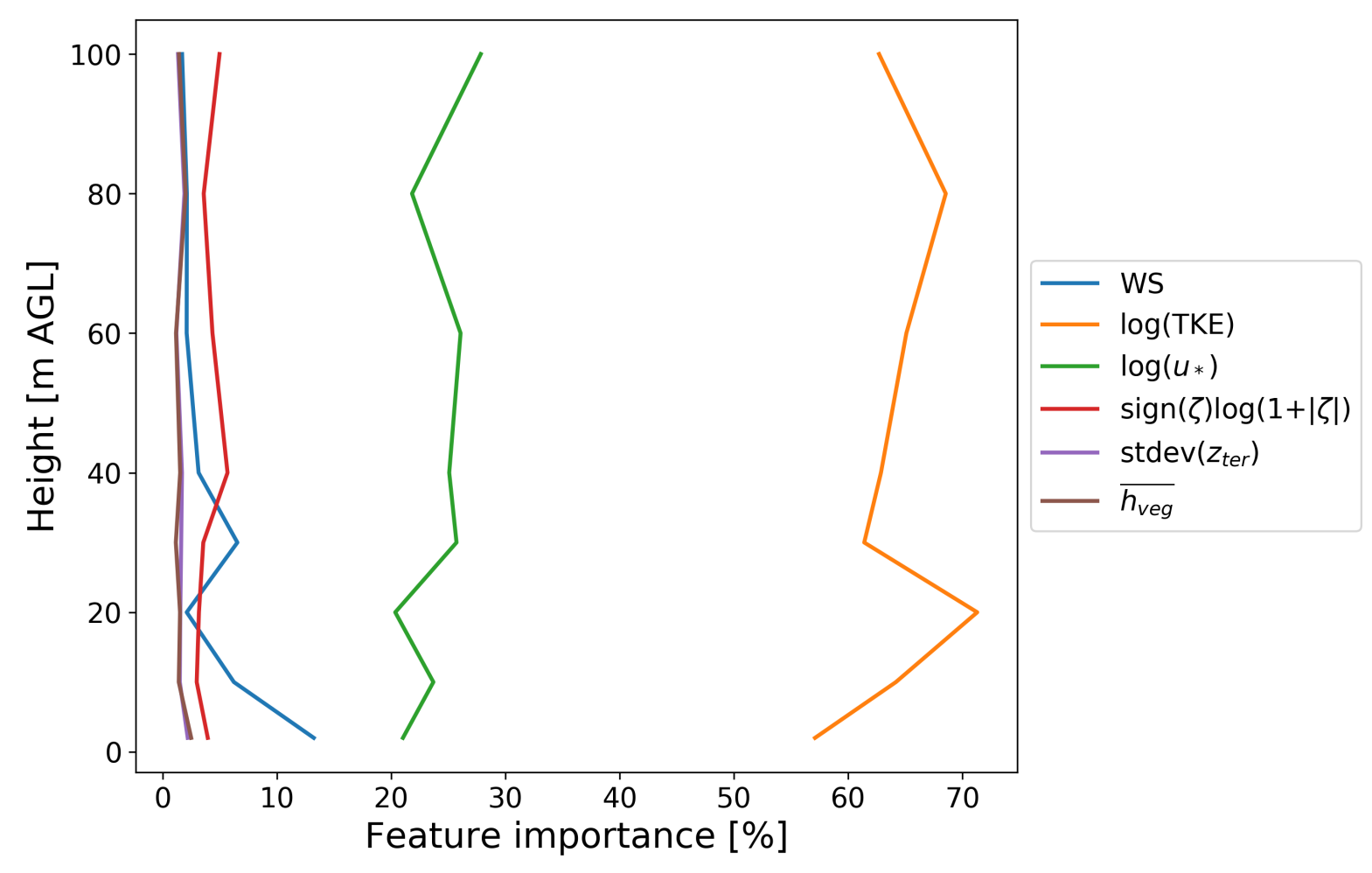

Figure S4: Variability of the random forest feature importance with height, derived from several random forests, each trained and tested with data from all the sonic anemometers at a single height only. 